\title{
Some Stable Explicit Difference Approximations to the Diffusion Equation
}

\author{
By Bert K. Larkin
}

1. Introduction. The use of digital computing machines in finding approximate solutions of partial differential equations is extensive. Because of expense in using such machines, there is considerable motivation to find the most efficient means of solution. It is the purpose of this communication to show that the difference scheme of Saul'ev [1], [2], [3], [4] may be extended to two or three dimensions. This technique for approximating the diffusion equation is explicit, stable for time steps of any size, and appears to be competitive with other methods of approximating the diffusion equation.

Consider the one-dimensional form of the diffusion equation in Cartesian coordinates. If the time derivative is approximated by a forward difference and the distance derivative is approximated by a central difference at the original time level, the system is explicit. That is, the dependent variable at the succeeding time step, may be written explicitly in terms of that variable at the earlier time step. It is well known [5] that stability requirements limit the size of the time step in proportion to the square of the size of the distance increment. For many applications the allowable time step is prohibitively small. By employing implicit methods, this limitation is removed [5]. In these methods the distance derivative is approximated by a central difference formula, but evaluated at the succeeding time step. Solution of simultaneous equations is necessary to establish values of the dependent variable at the new time level. It appears that the scheme of Saul'ev combines the best features of the two methods mentioned above. It avoids solving simultaneous equations but retains stability for any size time step.

For simplicity, the method of Saul'ev will be applied to the diffusion equation

$$
\begin{aligned}
\frac{\partial u}{\partial t} & =\frac{\partial^{2} u}{\partial x^{2}}+\frac{\partial^{2} u}{\partial y^{2}} \\
u(x, y, 0) & =f(x, y)
\end{aligned}
$$

where $f(x, y)$ has a valid Fourier series expansion in $x$ and $y$. The independent variables will be limited to $0 \leqq x \leqq x_{1}, 0 \leqq y \leqq y_{1}$, and $0 \leqq t$. Boundary conditions are assumed as

$$
\begin{aligned}
u(0, y, t) & =u(x, 0, t)=0 \\
u\left(x_{1}, y, t\right) & =u\left(x, y_{1}, t\right)=0 .
\end{aligned}
$$

Fourier series methods may be employed in solving the problem, and the exact solution may be deduced from that given by Carslaw and Jaeger [6, p. 187]. It is obvious that the method of Saul'ev is applicable to a more extensive class of problems. However, the object is to illustrate the difference scheme and show a com-

Received April 2, 1963, revised August 6, 1963. 
parison with other numerical methods. Extension to three-dimensions is possible. For the sake of simplicity two-dimensional problems will be treated.

2. Some Difference Schemes. Dependent variables, $v$ and $z$ will be employed in the difference scheme. Numbers $l, m, n$ are used to identify points in the $x, y, t$ space, and, hopefully, the difference scheme will be consistent with the diffusion equation such that

$$
\begin{aligned}
& v_{l, m, n} \approx u(l \Delta x, m \Delta y, n \Delta t) \\
& z_{l, m, n} \approx u(l \Delta x, m \Delta y, n \Delta t) .
\end{aligned}
$$

Numbers $l, m, n$ are integers restricted as follows:

$$
\begin{aligned}
& 0 \leqq l \leqq x_{1} / \Delta x \\
& 0 \leqq m \leqq y_{1} / \Delta y \\
& 0 \leqq n .
\end{aligned}
$$

Use of a difference operator is convenient. Let $\Delta_{l, n}(v)_{m}$ be defined as

$$
\Delta_{l, n}(v)_{m}=v_{l, m+1, n}-v_{l, m, n} .
$$

The two subscripts on $\Delta$ are constant in forming differences of $v$. Then difference quotient operators $L, R, P$ and $Q$ are defined as

$$
\begin{aligned}
& L(v)=\frac{\Delta_{l, m}(v)_{n}}{\Delta t}-\frac{\Delta_{m, n}(v)_{l}-\Delta_{m, n+1}(v)_{l-1}}{(\Delta x)^{2}}-\frac{\Delta_{l, n}(v)_{m}-\Delta_{l, n+1}(v)_{m-1}}{(\Delta y)^{2}} \\
& R(z)=\frac{\Delta_{l, m}(z)_{n}}{\Delta t}-\frac{\Delta_{m, n+1}(z)_{l}-\Delta_{m, n}(z)_{l-1}}{(\Delta x)^{2}}-\frac{\Delta_{l, n+1}(z)_{m}-\Delta_{l, n}(z)_{m-1}}{(\Delta y)^{2}} \\
& P(v)=\frac{\Delta_{l, m}(v)_{n}}{\Delta t}-\frac{\Delta_{m, n}(v)_{l}-\Delta_{m, n+1}(v)_{l-1}}{(\Delta x)^{2}}-\frac{\Delta_{l, n+1}(v)_{m}-\Delta_{l, n}(v)_{m-1}}{(\Delta y)^{2}} \\
& Q(z)=\frac{\Delta_{l, m}(z)_{n}}{\Delta t}-\frac{\Delta_{m, n+1}(z)_{l}-\Delta_{m, n}(z)_{l-1}}{(\Delta x)^{2}}-\frac{\Delta_{l, n}(z)_{m}-\Delta_{l, n+1}(z)_{m-1}}{(\Delta y)^{2}}
\end{aligned}
$$

By setting equations 2.7 through 2.10 to zero, the following equations may be obtained:

$$
\begin{aligned}
\left(1+w+c^{2}\right) v_{l, m, n+1}= & v_{l-1, m, n+1}+v_{l+1, m, n} \\
& -\left(1-w+c^{2}\right) v_{l, m, n}+c^{2}\left[v_{l, m-1, n+1}+v_{l, m+1, n}\right] \\
\left(1+w+c^{2}\right) z_{l, m, n\lrcorner}= & z_{l-1, m, n}+z_{l+1, m, n+1} \\
& -\left(1-w+c^{2}\right) z_{l, m, n}+c^{2}\left[z_{l, m+1, n+1}+z_{l, m-1, n}\right] \\
\left(1+w+c^{2}\right) v_{l, m, n+1}= & v_{l-1, m, n+1}+v_{l+1, m, n} \\
& -\left(1-w+c^{2}\right) v_{l, m, n}+c^{2}\left[v_{l, m-1, n}+v_{l, m+1, n+1}\right] \\
\left(1+w+c^{2}\right) v_{l, m, n+1}= & v_{l-1, m, n}+v_{l+1, m, n+1} \\
& -\left(1-w+c^{2}\right) v_{l, m, n}+c^{2}\left[z_{l, m-1, n+1}+z_{l, m+1, n}\right] .
\end{aligned}
$$


Constants $c$ and $w$ have been used such that

$$
\begin{aligned}
c & =\Delta x / \Delta y \\
w & =(\Delta x)^{2} / \Delta t .
\end{aligned}
$$

Either equation $2.11,2.12,2.13$, or 2.14 may be used to generate approximate solutions to the diffusion equation. Combinations of these equations also offer potentially interesting approximations. Of the possible computing schemes the following are considered in illustrative problems below:

1. Use equation 2.11 only.

2. Use equation 2.12 only.

3. Use equations 2.11 and 2.12 alternately. For example, assume $n$ in equation 2.11 is an even integer, and for all possible $l$ and $m, v_{l, m, n+1}$ is computed using equation 2.11. Modify equation 2.12 by adding one to the time level subscripts. Set $z_{l, m, n+1}$ in the modified equation 2.12 equal to $v_{l, m, n+1}$ and then compute $z_{l, m, n+2}$. By repetition of this sequence, the dependent variable is computed at odd time levels using equation 2.11 and at even time levels using modified equation 2.12. This corresponds to the scheme proposed by Saul'ev and may be termed an alternating direction explicit method.

4. Use both equations 2.11 and 2.12 at each time level and average the results. That is

$$
r_{l, m, n+1}=\frac{v_{l, m, n+1}+z_{l, m, n+1}}{2}
$$

The variables on the right side of equation 2.11 and 2.12 at time level $n$ are replaced by $r$ 's.

5. Use equations 2.13 and 2.14 alternately in the same manner as described under scheme 3. For example, equation 2.13 may be used on odd time levels and equation 2.14 may be used on even time levels. It may be considered that schemes 3 and 5 differ only in the direction of computation. Suppose computations are made on a square in the first quadrant of a two-dimensional space. Computations, using scheme 3, proceed from southwest to northeast, then from northeast to southwest, etc. Using scheme 5, computations proceed from northwest to southeast, then from southeast to northwest, etc.

3. Stability. Stability requirements simply imply that errors present at any stage of the computation do not grow in successive levels of computation. Reference [2] gives a stability condition which is more restrictive than necessary, $w \geqq \frac{1}{2}$. The correct stability condition, $w>0$, is shown in reference [3]. Use of the von Neumann method [7], [8] gives the stability condition, $w>0$, for the schemes listed above. The von Neumann method assumes that a set of errors $\epsilon_{l, m, n}$ exist at time level $n$ and this error may be expanded in a finite trigonometric series

$$
\epsilon_{l, m, n}=\sum_{h} \sum_{j} A_{h, j, n} e^{i l \alpha} e^{i m \beta}
$$

where $\alpha \equiv \frac{h \pi \Delta x}{x_{1}}, \beta \equiv \frac{j \pi \Delta y}{y_{1}}$. O'Brien [7] shows how coefficients $A$ in equation 3.1 may be computed, but the computation is not required here. 
Stability implies that the absolute value of $A_{h, j, n+1} / A_{h, j, n}$ cannot be greater than unity for all possible $h, j, n$. By substituting the general term of equation 3.1 into equation 2.11 cancelling common factors and solving for the ratio of successive $A$ coefficients, one obtains

$$
\frac{A_{h, j, n+1}}{A_{h, j, n}}=\frac{w-1-c^{2}+e^{i \alpha}+c^{2} e^{i \beta}}{w+1+c^{2}-e^{-i \alpha}-c^{2} e^{-i \beta}} .
$$

Call the numerator and denominator of the right side of equation $3.2 N$ and $D$ respectively. The imaginary parts of $N$ and $D$ are identical. Thus the absolute value of $N / D$ will be less than or equal to unity provided the following condition is satisfied

$$
[\operatorname{Re}(D)]^{2}-[\operatorname{Re}(N)]^{2} \geqq 0
$$

By expansion of $N$ and $D$ it follows that

$$
[\operatorname{Re}(D)]^{2}-[\operatorname{Re}(N)]^{2}=4 w\left[1-\cos \alpha+c^{2}(1-\cos \beta)\right] .
$$

This obviously satisfies condition 3.3 for all $w>0$. Thus the difference scheme given by equation 2.11 is stable for positive time increments of any magnitude.

Performing similar analyses on equations $2.12,2.13$, and 2.14 shows that these difference schemes are stable for time increments of any size.

4. Truncation Errors. An acceptable difference scheme must converge to the partial differential equation as increment sizes of the independent variables vanish. This property is called consistency and it requires that the truncation error, defined below, vanish as the mesh size vanishes. Operator $L$ has a trunction error given by

$$
E L=\left|L(v)-\left(\frac{\partial v}{\partial t}-\frac{\partial^{2} v}{\partial x^{2}}\right)_{l, n}\right| .
$$

For simplicity only the one-dimensional case will be treated. Error terms in $\Delta y$ will be of the same form as those in $\Delta x$ and the following results may be easily expanded to two space dimensions. By virtue of this simplification, equations 2.7 and 2.9 are identical as are equations 2.8 and 2.10. Thus, it is only necessary to consider consistency of the $L$ and $R$ operators with the diffusion equation. Consistency of both $L$ and $R$ operators with the diffusion equation is sufficient to guarantee consistency of all the difference schemes listed above. The analysis below follows the arguments of Richtmyer [5, p. 18]. Assume $v$ and $z$ are continuous functions of $x, t$ and have Taylor series expansions, through fourth order terms. Difference quotients in equations 2.7 are as follows

$$
\begin{aligned}
\frac{v_{l+1, n}-v_{l, n}}{(\Delta x)^{2}}=(\Delta x)^{-1} \frac{\partial v}{\partial x}+ & \frac{1}{2} \frac{\partial^{2} v}{\partial x^{2}}+\frac{\Delta x}{6} \frac{\partial^{3} v}{\partial x^{3}}+\cdots \\
\frac{v_{l, n+1}-v_{l-1, n+1}}{(\Delta x)^{2}}=(\Delta x)^{-1} \frac{\partial v}{\partial x} & -\frac{1}{2} \frac{\partial^{2} v}{\partial x^{2}}+\left(\frac{\Delta t}{\Delta x}\right) \frac{\partial^{2} v}{\partial x \partial t}+\frac{\Delta x}{6} \frac{\partial^{3} v}{\partial x^{3}} \\
& -\frac{\Delta t}{2} \frac{\partial^{3} v}{\partial t \partial x^{2}}+\frac{(\Delta t)^{2}}{2 \Delta x} \frac{\partial^{3} v}{\partial t^{2} \partial x}+\frac{(\Delta t)^{3}}{6(\Delta x)^{2}} \frac{\partial^{3} v}{\partial t^{3}}+\cdots \\
\frac{v_{l, n+1}-v_{l, n}}{\Delta t}=\frac{\partial v}{\partial t}+\frac{\Delta t}{2} \frac{\partial^{2} v}{\partial t}+ & +\frac{(\Delta t)^{2}}{6} \frac{\partial^{3} v}{\partial t^{3}}+\cdots
\end{aligned}
$$


Add equation 4.4 and 4.3 and subtract equation 4.2 to obtain

$$
\begin{gathered}
L(v)=\frac{\partial v}{\partial t}-\frac{\partial^{2} v}{\partial x^{2}}+\frac{\Delta t}{2}\left(\frac{\partial^{2} v}{\partial t^{2}}-\frac{\partial^{3} v}{\partial t \partial x^{2}}\right)+\left(\frac{\Delta t}{\Delta x}\right) \frac{\partial^{2} v}{\partial x \partial t}+\frac{(\Delta t)^{2}}{2 \Delta x} \frac{\partial^{3} v}{\partial x \partial t^{2}}+\cdots \\
E L=\left|\left(\frac{\Delta t}{\Delta x}\right) \frac{\partial^{2} v}{\partial x \partial t}+\frac{\Delta t}{2}\left(\frac{\partial^{2} v}{\partial t^{2}}-\frac{\partial^{3} v}{\partial t \partial x^{2}}\right)+\frac{(\Delta t)^{2}}{2 \Delta x} \frac{\partial^{3} v}{\partial x \partial t^{2}}+\cdots\right|
\end{gathered}
$$

Derivatives above are taken at $l \Delta x, n \Delta t$. If $v$ satisfies the diffusion equation, the second term of the $E L$ expansion vanishes. In the above development the fourth order terms have been dropped. Consistency of the $L$ operator with the diffusion equation requires that $\Delta t / \Delta x \rightarrow 0$ as $\Delta x \rightarrow 0$. By diminishing $\Delta x$ and $\Delta t$ such that $w$ is constant, the leading term of equation 4.6 would be of first degree in $\Delta x$, all other terms being of higher order. By a similar procedure $E R$, the truncation error for operator $R$ is

$$
E R=\left|-\left(\frac{\Delta t}{\Delta x}\right) \frac{\partial^{2} z}{\partial x \partial t}+\frac{\Delta t}{2}\left(\frac{\partial^{2} z}{\partial t^{2}}-\frac{\partial^{3} z}{\partial t \partial x^{2}}\right)-\frac{(\Delta t)^{2}}{2 \Delta x} \frac{\partial^{3} z}{\partial x \partial t^{2}}+\cdots\right| .
$$

Consistency of the $R$ operator will be satisfied by setting $\Delta x$ and $\Delta t$ to zero such that $w$ is constant. This procedure of using constant $w$ is sufficient to guarantee consistency of all difference schemes listed above. Because the leading terms of 4.6 and 4.7 have opposite signs, averaging results of $L$ and $R$ operators, scheme 4 , has a truncation error of second degree.

In applications, scheme 3, an alternating direction explicit method, seems to yield much more favorable truncation errors than either schemes 1 or 2 . Errors introduced by operator $L$ appear to be partially compensated by using operator $R$ in the following time step. The same results hold for scheme 5 .

5. Some Numerical Examples. As a simple example, the following problem was treated

$$
\begin{aligned}
\frac{\partial u}{\partial t} & =\frac{\partial^{2} u}{\partial x^{2}} \\
u(x, 0) & =1 \\
u(0, t) & =0 \\
u(1, t) & =1 .
\end{aligned}
$$

The exact solution, from Carslaw and Jaeger [6, p. 100] was programmed for a Burroughs 205 computer. Listed in Table 1 are errors, $v-u$, for the point $x=0.5$, $t=0.16$. Uniform distance increments of 0.1 and uniform time steps shown below were used. From the exact solution, $u$ was computed as 0.63124 . The implicit scheme used is the conventional $T$ form, see p. 93, $\$ 2$, of Richtmyer [5].

Note that the errors corresponding to schemes 1 and 2 vary almost linearly with $\Delta t$. Scheme 1 gives errors which are negative and scheme 2 gives errors which are positive. These results support predictions of equations 4.6 and 4.7. Both schemes 3 and 4 give errors of smaller magnitude than corresponding errors of schemes 1 or 2 . Of the schemes listed, scheme 3 is preferred since it is more accurate and requires less computer time. Scheme 3 required $40 \%$ as much computing time as did the implicit method. 
TABLE 1

Error Tabulation For One-Dimensional Example

\begin{tabular}{l|r|r|r}
\hline \multirow{2}{*}{ Method } & \multicolumn{3}{|c}{ Time increment } \\
\cline { 2 - 4 } & \multicolumn{1}{|c|}{0.005} & \multicolumn{1}{|c}{0.01} & \multicolumn{1}{|c}{0.02} \\
\hline Implicit & 0.0056 & 0.0104 & 0.0197 \\
Scheme 1 & -0.0122 & -0.0250 & -0.0516 \\
Scheme 2 & 0.0137 & 0.0276 & 0.0609 \\
Scheme 3 (using $L$ first) & 0.0020 & 0.0058 & 0.0133 \\
Scheme 4 & 0.0031 & 0.0093 & 0.0268 \\
\hline
\end{tabular}

TABLE 2

Error Tabulation for Two-Dimensional Example

\begin{tabular}{|c|c|c|c|c|}
\hline \multirow{2}{*}{ Scheme } & \multicolumn{4}{|c|}{$\Delta t$} \\
\hline & 0.02 & 0.01 & 0.005 & 0.0025 \\
\hline $\begin{array}{l}3 \text { starting northeast } \\
5 \text { starting northwest } \\
\text { alternating direction implicit }\end{array}$ & $\begin{array}{r}-0.0255 \\
-0.0132 \\
0.0032\end{array}$ & $\begin{array}{r}0.0012 \\
0.0011 \\
-0.0039\end{array}$ & $\begin{array}{r}0.0000 \\
-0.0002 \\
-0.0021\end{array}$ & $\begin{array}{l}-0.0013 \\
-0.0014 \\
-0.0019\end{array}$ \\
\hline
\end{tabular}

The following two dimensional problem was considered

$$
\begin{aligned}
\frac{\partial u}{\partial t} & =\frac{\partial^{2} u}{\partial x^{2}}+\frac{\partial^{2} u}{\partial y^{2}} \\
u(x, y, 0) & =1 \\
u(1, y, t) & =u(x, 1, t)=0 \\
\left.\frac{\partial u}{\partial x}\right|_{0, y, t} & =\left.\frac{\partial u}{\partial y}\right|_{x, 0, t}=0 .
\end{aligned}
$$

This is the same problem treated by Peaceman and Rachford [8]. Computation using the exact solution gave a value of 0.62177 at $x=0.5, y=0.5, t=0.08$. Using uniform distance increments of 0.1 and uniform time steps, numerical estimates were obtained. The errors presented in Table 2 are the numerical solution minus the exact solution. The alternating direction implicit method is that described in [8] and the method employed to solve the simultaneous equations was that given in [8]. For this problem schemes 3 and 5 gave equivalent accuracy and both compared favorably with the alternating direction implicit method. Especially interesting is the fact that schemes 3 and 5 required about $20 \%$ as much computing time as did the implicit method.

6. Conclusion. The alternating direction explicit method for generating numerical solutions to the diffusion equation is stable for time increments of any magnitude. Because it is an explicit method, it holds a speed advantage over implicit methods for computations over a single time level. The above example 
problems suggest that there is no appreciable loss of accuracy by using the alternating direction explicit method instead of an implicit method.

7228 South Grant

Littleton, Colorado

1. J. H. Giese, Numerical Analysis, "Recent Soviet contributions to mathematics." The Macmillan Co., New York, 1962, p. 86-88.

2. V. K. SAUL'Ev, "A method of numerical solution for the diffusion equation," Dokl. Akad. Nauk SSSR (NS), 115 (1957), 1077-1079. RZh (1958), 6198.

3. V. K. SAUL'Ev, "Numerical integration of parabolic equations," Dokl. Akad Nauk SSSR (NS), 117 (1957), 36-39. MR, 20, 2847; RZh (1958), 9251.

4. V. K. SAUL'Ev, "Methods of increased accuracy and two-dimensional approximations to solutions of parabolic equations," Dokl. Akad. Nauk SSSR (NS), 118 (1958), 1088-1090. RZh (1959), 869.

5. Robert D. Richtmyer, Difference Methods for Initial-Value Problems. Interscience Publishers, Inc., New York, 1957.

6. H. S. Carslaw, \& J. C., JAeger, Conduction of Heat in Solids. 2nd ed., Oxford University Press, 1959.

7. G. G. O'Brien, M. A. Hyman, \& S., Kaplan, "A study of the numerical solution of partial differential equations," J. Math. Phys., v. 29, 1950, p. 223-251.

8. D. W. PEACEMAN, \& H. H. RACHFORD, "The solution of parabolic and elliptic differential equations," J. Soc. Indust. Appl. Math., vol. 3, 1955, p. 28-41. 